\title{
The Long-Term Outcome of Cyberknife Therapy for Head and Neck Paraganglioma: Management Suggestion From a Single Center Experience
}

Sukwoo Hong ( $\nabla$ honsohkaisei6031@gmail.com )

Japanese Red Cross Medical Center https://orcid.org/0000-0002-3664-9245

Kenji Kagawa

Japanese Red Cross Medical Center

Kengo Sato

Japanese Red Cross Medical Center

Shunsuke Ichi

Japanese Red Cross Medical Center

\section{Clinical Study}

Keywords: CyberKnife, glomus, hormone, functional

Posted Date: February 1st, 2021

DOI: https://doi.org/10.21203/rs.3.rs-163980/v1

License: (c) (i) This work is licensed under a Creative Commons Attribution 4.0 International License. Read Full License 


\section{Abstract}

Introduction

To analyze the long-term follow-up data of CyberKnife treatment (CKT) for head and neck paragangliomas (HNPGs).

Methods

Patients who received CKT to HNPGs from 2010 to 2019 were retrospectively reviewed.

Results

A total of 34 HNPGs from 29 patients were identified. The mean age was $50( \pm 16)$ years old, and 15 patients $(52 \%)$ were female. Previous operation was done in 15 patients $(55 \%)$. Four cases $(14 \%)$ were functional in hormone production. According to Fisch classification, one case (3\%) was B, $12(42 \%)$ were $C, 14(48 \%)$ were $D$, and two cases $(7 \%)$ were out of classification. The median prescribed dose covered $95 \%$ of the planning target volume was 2500 (IQR $2100-2600$ ) cGy and the median target volume was 10 (IQR $5.8-21.3) \mathrm{cm}^{3}$. The local control rate was $97 \%$. The median progression-free survival was 66 (IQR 27.5 - 95.0) months and $93.8 \%$, and $91.7 \%$ were free of tumor progression at five, and seven years respectively. During the follow-up, one case (3\%) resulted in permanent facial nerve palsy (CTCAE 1 , House-Brackmann grade II), and another case (3\%) resulted in asymptomatic cerebellar radiation necrosis. Univariate and multivariate analysis showed no previous surgical history (OR 10.3, 95\% $\mathrm{Cl} 1.5-$ $69.3, p=0.02$ ) was a positive predictor of symptom improvement. We devised a treatment flow-chart based on our finding.

Conclusions

CKT for HNPGs was an effective treatment with little side effect over the long term and should be considered the first-line therapy for symptomatic nonfunctional HNPGs for better symptom control.

\section{Introduction}

Paragangliomas, also known as glomus tumors, in the head and neck may be difficult to treat.[1, 2] They are WHO grade I tumor, and commonly arise in the jugulotympanic regions[3], making it difficult to perform complete surgical excision with leaving the surrounding nerves and vessels intact.[4] A strong tumor enhancement on computed tomography and magnetic resonance image (MRI) helps make the diagnosis and hypervascularity on digital subtraction angiography confirms the diagnosis in difficult cases. $[5,6]$ Due to their origin in neural crest cells associated with autonomic ganglia, the jugulotympanic paragangliomas may secrete catecholamine, in which case they are called functional paragangliomas.[3] Most skull base paragangliomas are treated by surgical resection, endovascular embolization, radiation, and their combination. The two main kinds of stereotactic radiosurgery or radiotherapy are gamma knife, and CyberKnife. Compared to gamma knife, CyberKnife outcome has not been published as much.[7, 8] 
To further accumulate long-term evidence of CyberKnife therapy (CKT) on head and neck paragangliomas (HNPGs), we analyzed the outcome of cases from our center. Since no treatment guidelines for HNPGs exist[8], we aimed to establish our own management flow-chart if we found anything meaningful from the analysis.

\section{Methods}

Patients who received CKT for HNPGs in the period from 2010 to 2019 were included and various data were recorded including age, sex, signs and symptoms before CKT, side of radiation, history of previous operation or radiation, functionality in hormone production by the tumor, the number of tumors, Fisch classification[9], form of treatment (primary, adjuvant, or secondary for relapsed cases), parameters for CKT, radiological response, changes in symptoms, adverse radiation effect (ARE), and the length of follow-up among others. The diagnosis of HNPGs was made radiologically by MRI with or without DSA for non-operated patients, and further confirmed pathologically for operated patients. Based on the magnetic resonance image, we defined the response to CKT into three categories of partial response (PR), sustained disease (SD), and progressive disease (PD). ARE was evaluated by Common Terminology Criteria for Adverse Events (CTCAE) if listed. From the acquired data, univariate and multivariate analyses were performed to identify prognostic factors for tumor progression and symptoms improvement. The factors whose $p$ value less than 0.1 from the univariate analysis were considered for multivariate analysis. Multicollinearity test was done to identify factors to exclude in multivariate analysis. Progression free survival (PFS) and overall survival (OS) curves were drawn.

Statistical analysis was done using SPSS version 25.0 (IBM Inc., Armonk, NY, USA). The values were listed as means for parametric data, and median for nonparametric data. Shapiro-Wilk test was used to tell parametric from nonparametric data. Binary logistic regression was used to evaluate the prognostic factors for radiological outcome and symptom improvement. Kaplan-Meier method was used to draw PFS and OS curves from the last day of CKT. P value of 0.05 or less was considered statistically significant.

Our institutional review board did not require informed consent for study participation because this study relied on information obtained as part of routine clinical practice.

\section{Results}

A total of 29 patients and 34 tumors were identified (Table 1). Female constituted $52 \%$ of the patients. The common symptoms were hearing impairment (52\%), tinnitus (52\%), hoarseness (42\%), and dysphagia (34\%). Previous operation were performed in 15 cases (52\%). Four cases (14\%) were functional in catecholamine production by tumors, and all underwent surgical resection before CKT. Most of the tumors were Fish classification[9] D1 or D2 (14 cases, 48\%) at the time of CKT. Two cases were not classified according to Fisch classification, as they were neither glomus jugulare or tympanicum. The most common form of CKT was primary treatment (14 cases, $48 \%$ ), followed by treatment for relapsed 
tumors (12 cases, $42 \%$ ) and adjuvant treatment (3 cases, 10\%). The median time between the previous surgery and CKT was 25 months and that for relapsed tumors was about 40 months (Table 1). The parameters of CKT is summarized in the Table 2. All therapy were done in multisession and the median target tumor volume was $10(5.8-21.3) \mathrm{cc}$. 
Table 1

Summary of the baseline patient characteristics

\begin{tabular}{|c|c|}
\hline Total & 29 \\
\hline Age (mean) (yr) & $50 \pm 16$ \\
\hline Female & $15(52 \%)$ \\
\hline Symptoms & $6(21 \%)$ \\
\hline Facial palsy & $3(10 \%)$ \\
\hline Occasional facial spasm & $2(7 \%)$ \\
\hline Dysgeusia & $15(52 \%)$ \\
\hline Hearing impairment & $15(52 \%)$ \\
\hline Tinnitus & $4(14 \%)$ \\
\hline Dizziness & $12(42 \%)$ \\
\hline Hoarseness & $10(34 \%)$ \\
\hline Dysphagia & $2(7 \%)$ \\
\hline \multicolumn{2}{|l|}{ Dysarthria } \\
\hline Right side of radiation & $16(55 \%)$ \\
\hline Previous operation & $15(52 \%)$ \\
\hline Previous radiation & $2(7 \%)$ \\
\hline Hormonally functional & $4(14 \%)$ \\
\hline Multiple lesions in the head and neck & $2(7 \%)$ \\
\hline Fisch classification & $1(3 \%)$ \\
\hline B & $12(42 \%)$ \\
\hline C & $14(48 \%)$ \\
\hline D & $2(7 \%)$ \\
\hline \multicolumn{2}{|l|}{ Others } \\
\hline Form of CKT & $14(48 \%)$ \\
\hline Primary Tx & $3(10 \%)$ \\
\hline Adjuvant Tx & $12(42 \%)$ \\
\hline Tx for relapsed tumor & \\
\hline
\end{tabular}




\begin{tabular}{|ll|}
\hline Total & 29 \\
\hline Time from the last surgery to relapse (mos) (median) (IQR) & $37.0(8.0-72.5)$ \\
Time from the surgery to CKT (mos) (median) (IQR) & $25(8-56)$ \\
Adjuvant Tx (mos) (median) (IQR) & $4(3.5-8.0)$ \\
Tx for relapsed tumor (mos) (median) (IQR) & $40.5(10.0-83.0)$ \\
\hline
\end{tabular}

Table 2

Summary of CyberKnife therapy

\begin{tabular}{ll|} 
D95 (median) (IQR) (cGy) & $2500(2100-2600)$ \\
Fraction (median) (IQR) & $5(3-5)$ \\
Target (tumor) volume (median) (IQR) $\left(\mathrm{cm}^{3}\right)$ & $10(5.8-21.3)$ \\
\hline
\end{tabular}

The outcome of CyberKnife therapy is summarized in the Table 3. Local control (PR + SD) was achieved in $97 \%$. The median time to confirm radiological PR was 12 (IQR 6-19) months. One PD case (3\%) deteriorated due to metastasis to a thoracic vertebral body 17 months following the last CKT, which required an emergent surgery. He committed suicide 59 months after the last CKT, though the tumor treated with CyberKnife was controlled. The death was not directly due to the tumor. As for the symptom control, $41 \%$ of patients had some improvement. The likely symptoms to improve were tinnitus (47\%), and hearing impairment (33\%). On the other hand, no one had any subjective improvement in facial palsy, dysgeusia, or dysarthria. As for the ARE, one case (3\%) resulted in asymptomatic radiation necrosis in the cerebellar hemisphere near the radiated region (CTCAE grade 1), and another one (3\%) resulted in facial nerve disorder (CTCAE grade 1, House-Brackmann grade II). Although ARE occurred in two cases (7\%), only one (3\%) of them (facial nerve disorder) was clinically significant. It occurred in a 28-year-old male patient with Fisch class B tumor. The D95 was 2100 cGy in 3 fraction for 0.7 cc target tumor volume. 
Table 3

Outcome after CyberKnife therapy

\begin{tabular}{|c|c|}
\hline Radiological outcome & $15(52 \%), 12(6-19)$ months \\
\hline PR, median time from the last CKT (IQR) & $13(45 \%)$ \\
\hline SD & $1(3 \%), 17$ months \\
\hline \multicolumn{2}{|l|}{ PD, time from the last CKT } \\
\hline The number of patients whose symptoms improved & $12(41 \%)$ \\
\hline Occasional facial spasm & $1(33 \%)$ \\
\hline Hearing impairment & $5(33 \%)$ \\
\hline Tinnitus & $7(47 \%)$ \\
\hline Dizziness & $1(25 \%)$ \\
\hline Hoarseness & $2(17 \%)$ \\
\hline Dysphagia & $1(10 \%)$ \\
\hline Adverse radiation effect & $1(3 \%)$ \\
\hline Facial nerve disorder* & $1(3 \%)$ \\
\hline Asymptomatic cerebellar necrosis ${ }^{\star \star}$ & $1(3 \%)$ \\
\hline \multicolumn{2}{|l|}{ Dead*** } \\
\hline PFS (median) (IQR) (months) & $66.0(27.5-95.0)$ \\
\hline OS (median) (IQR) (months) & $66.0(28.0-95.0)$ \\
\hline \multicolumn{2}{|l|}{ *CTCAE grade 1, House-Brackmann grade II } \\
\hline \multicolumn{2}{|c|}{ **Small radiation necrosis in the right cerebellar hemisphere near the radiated region } \\
\hline$\star \star \star$ Due to suicide & \\
\hline
\end{tabular}

The medians of progression-free survival and overall survival were both 66 months. PFS and OS curves were drawn in the Fig. 1. PFS at 3, 5, and 7 years were $94.1 \%, 93.8 \%$, and $91.7 \%$ respectively. We illustrate two PR cases as examples (Fig. 2).

\section{Analysis for prognostic factors}

Univariate analysis for the symptoms improvement showed that female sex, and having no history of previous surgery were statistically significant factors for recovery of symptoms (Table 4). The status of operation ( $p=0.01)$ and form of treatment $(p=0.06)$ were statistically correlated (correlation coefficient of 0.84 ). Consequently, the sex (female) and the status of having no previous operation were included in the multivariate analysis, the result of which is summarized in the Table 4. The status of no history of 
surgery was still significant $(p=0.02)$. The same kind of analysis was performed exclusively in the nonfunctional group (no hormone production by the tumor). The same two factors (female sex, and no history of previous operation) showed a tendency $(p=0.06)$ for symptom improvement. Univariate analysis for the PD (local control failure) resulted in no variables (age, sex, previous operation, time to $\mathrm{CKT}$, target volume, and firm of CKT) to be statistically significant (not shown).

Table 4

Univariate and multivariate analyses on the improvement of overall symptoms.

\begin{tabular}{|c|c|c|c|c|}
\hline & \multicolumn{2}{|c|}{ Overall improvement } & \multicolumn{2}{|c|}{$\begin{array}{l}\text { Overall improvement } \\
\text { (nonfunctional group) }\end{array}$} \\
\hline & $\begin{array}{l}\text { Univariate (p } \\
\text { value) }\end{array}$ & $\begin{array}{l}\text { Multivariate } \\
\text { (OR, 95\% Cl, p } \\
\text { value) }\end{array}$ & $\begin{array}{l}\text { Univariate ( } p \\
\text { value) }\end{array}$ & $\begin{array}{l}\text { Multivariate } \\
\text { (OR, 95\% Cl, p } \\
\text { value) }\end{array}$ \\
\hline Age & 0.21 & & 0.12 & \\
\hline Sex (female) & 0.04 & $\begin{array}{l}5.9,0.8-40.8 \\
0.07\end{array}$ & 0.03 & $\begin{array}{l}6.7,0.9-47.3 \\
0.06\end{array}$ \\
\hline Side of CKT & 0.22 & & 0.32 & \\
\hline $\begin{array}{l}\text { No previous } \\
\text { operation }\end{array}$ & 0.01 & $\begin{array}{l}10.3,1.5-69.3 \\
0.02\end{array}$ & 0.03 & $\begin{array}{l}6.7,0.9-47.3 \\
0.06\end{array}$ \\
\hline Previous radiation & 0.8 & & 0.95 & \\
\hline Functional & 1 & & - & \\
\hline $\begin{array}{l}\text { Fisch } \\
\text { classification }\end{array}$ & 0.32 & & 0.33 & \\
\hline Form of CKT & 0.06 & & 0.2 & \\
\hline Time to CKT & 0.99 & & 0.76 & \\
\hline $\begin{array}{l}\text { Target tumor } \\
\text { volume }\end{array}$ & 0.79 & & 0.98 & \\
\hline $\begin{array}{l}\text { Radiological } \\
\text { outcome }\end{array}$ & 0.81 & & 0.46 & \\
\hline
\end{tabular}

\section{Discussion}

In this study, we confirmed the long-term effectiveness of CKT to the HNPGs with acceptable rate of clinically significant adverse effects (3\%). We also found that those patients with no history of surgical excision of their HNPGs were more likely to have improvement in symptoms after CKT (Table 4). Although quite a few case series demonstrating the effects of gamma knife treatment to HNPGs exist[10-12, 2], CKT evidence for these tumors still need to be accumulated especially long-term 
results[8]. We believe that our series of 29 cases, although the number of cases are not as many as some previous studies, the relatively longer median follow-up period of 66 months ( 15 cases were followed for more than five years) adds further valuable data to the current literature.

\section{Radiological tumor control}

In the median follow-up of 66 months, 97\% (28/29) local control rate was attained and we observed one (3\%) PD at 17 months from the last CKT. On the other hand, radiological PR was observed between six to 19 months from the last CKT (Table 3). Based on this, radiological outcome after CKT may be more likely to be determined within the first two years. One thing should be mentioned to the PD case is that systemic screening for HNPGs metastasis was not done before CKT and that we are not sure that the case was truly PD. The radiated site was well controlled at the time of the latest radiological follow-up. Considering the fact that about $5 \%$ of HNPGs are associated with malignancy $[1,13]$, systemic screening should have been done at some around the CKT. No prognostic factors for PD were identified in our series as was the case with a similar past study.[14] This may be due to the small number of local control failure. The local control rate and the rate of ARE were in line with previous studies.[14, 8, 15] Our study adds to the evidence that CKT is effective to HNPGs. Although quite a few literature state the efficacy of radiation treatment to HNPGs, we do not have consensus guidelines for the treatment of HNPGs.[8] Several papers advocates the primary role of radiation treatment. $[15,16]$ However, all of these studies are retrospective ones, and we may need to have a randomized control trial comparing surgical resection with radiation treatment.

\section{Symptom improvement and adverse effects}

In our cohort, 41\% (12/29) experienced some degree of symptoms improvement. Although a little lower, the symptom improvement rate after CKT in our study was not much deviated from that of past studies $(48-56 \%) .[2,4,14,8]$ Hearing impairment (33\%) and tinnitus (47\%) were likely to improve than other symptoms (Table 3) and this tendency was supported by a recent meta-analysis.[4] Univariate and multivariate analysis revealed that the status of no past surgical resection was a significant prognostic factor for better clinical outcome (Table 4). The female sex showed tendency to have a better clinical outcome as well. When subgroup analyses was performed for nonfunctional group, the female sex and no history of surgery were still the two factors that showed tendency to have a better symptom control. This is probably that, by the surgical impact, the nerves are more damaged than relieved by surgical decompression. It is unclear why the female sex was more likely to respond clinically to CKT. It must be noted that, since not all the symptoms were objectively, or quantitatively evaluated pre-/ post- CKT, it is likely that some symptoms' improvement were susceptible to subjective feelings at the time of the followup. Nevertheless, it is the patients' satisfaction that counts in the real world, and even if the symptoms improvement was largely due to subjective feeling, the results are still applicable to the clinical practice to some extent. Having said that, females may be more likely to feel a slight change in symptoms, resulting in females sex being a good prognostic factor. It should be mentioned that these findings were not previously endorsed by any other studies and that this finding remains to be carefully evaluated in the 
future studies. However, some meta-analyses[16, 17] found that cranial nerve deficits were higher in the gross total resection group than radiation treatment group. This finding indirectly supports our finding that having no surgical history is a positive factor for symptom improvement. On the whole, to the best of our knowledge, no factors are consistently found to be a significant prognostic factor. One study showed that the female sex, right-sided tumor, primary radiation treatment, and hearing impairment symptoms to be negative prognostic factors for local control failure.[4] Another study found no significant prognostic factors for symptom improvement.[8] Other study showed that having cranial neuropathy before radiation treatment was a risk factor for lack of symptom improvement.[14]

As for ARE, the rate was 7\% (2/29), which is comparable to past similar study.[14] One was asymptomatic cerebellar radiation necrosis, the other one was facial nerve disorder (CTCAE grade 1). This facial palsy may be due to damage to the tympanic segment of the facial nerve. A recent metaanalysis revealed that CyberKnife was associated with the least likeliness of ARE compared to linear accelerator or gamma knife.[4]

\section{Management suggestion based on our series}

Based on our series, and its outcome described above, we made a flow-chart for HNPGs management (Fig. 3). Once paraganglioma is in the differential diagnosis and radiologically likely, determination should be made whether it is symptomatic or not. If asymptomatic, it may be waited and scanned for follow-up. In a study, $45 \%$ of tumors remained stable or regressed in size over more than five years followup.[18] On the other hand, treatment intervention should be considered for symptomatic ones based on its functionality. Since all four (14\%) of our functional HNPGs were treated by surgical resection and observed to find their recurrence at the mean follow-up of $16 \pm 15$ months, we suggest surgical resection of the symptomatic functional HNPGs followed by CKT. We think the postoperative CKT may be performed only after the diagnosis of recurrence (or relapse), or as adjuvant therapy. Surgical option should be reserved for rapidly growing and functional tumors.[19, 8] Lieberson et al. suggested a treatment algorism, which had something in common with ours.[19] As for the symptomatic nonfunctional HNPGs, we think CKT is the best primary treatment based on the analysis in the Table 3 for better clinical symptom control especially if female cases. It may be treated primarily by surgical resection followed by CKT soon after the surgery as adjuvant therapy or at the time of radiological relapse as secondary therapy. In either way, since no prognostic factors for PD were found in our series as well as past studies, radiological control would not be affected. After CKT, the first two years should be closely monitored radiologically (Table 3). Also systemic screening for metastasis of HNPGs is strongly considered if not already done. As for concurrently found multiple HNPGs, each lesion can be managed in the same manner. In our series, two patients (4\%) had more than one HNPG. We performed CKT for only one symptomatic lesion in both patients and followed the other non-radiated lesions, which did not grow for 31 and 61 months respectively for two patients. One of them strongly wished to have the remaining non-growing lesions to be treated, and we did CKT for these legions in the end.

\section{Limitations}


The limitations were retrospective nature and that this study was carried out in single institution. And there was not enough number of cases where PD was observed to detect prognostic factors if any.

\section{Conclusions}

CKT for HNPGs was an effective treatment option with little adverse effect over the long term. We suggested management algorisms based on our case series' data and recommend primary treatment with CKT for symptomatic nonfunctional HNPGs.

\section{Declarations}

Funding: Not applicable.

Conflicts of interest/Competing interests: Not applicable.

Availability of data and material: Data transparency was confirmed.

Code availability: Not applicable

Ethical approval: This study was done under our institutional review board approval and did not require patient consent.

Consent to participate: Our institutional review board did not require informed consent for study participation because this study relied on information obtained as part of routine clinical practice.

Consent for publication: Not applicable.

Authors' contributions: All authors read and approved the final manuscript. SH made a study design, collected patient data, drafted and revised the manuscript. KK made a contribution to revising the original draft. KS, and SI were the supervisors.

\section{References}

1. Forbes JA, Brock AA, Ghiassi M, Thompson RC, Haynes DS, Tsai BS (2012) Jugulotympanic paragangliomas: 75 years of evolution in understanding. Neurosurgical Focus FOC 33 (2):E13. doi:10.3171/2012.6.Focus12138

2. Tripathi M, Rekhapalli R, Batish A, Kumar N, Oinam AS, Ahuja CK, Deora H, Aggarwal A, Mohindra S, Kaur P, Kaur R, Bhatt S, Gurnani J (2019) Safety and Efficacy of Primary Multisession Dose Fractionated Gamma Knife Radiosurgery for Jugular Paragangliomas. World Neurosurg 131:e136e148. doi:10.1016/j.wneu.2019.07.090

3. Louis DN, Wiestler OD, Ohgaki H, Cancer IAfRo (2016) WHO Classification of Tumours of the Central Nervous System. vol v. 1. International Agency for Research on Cancer, 
4. Fatima N, Pollom E, Soltys S, Chang SD, Meola A (2020) Stereotactic radiosurgery for head and neck paragangliomas: a systematic review and meta-analysis. Neurosurgical review. doi:10.1007/s10143020-01292-5

5. Reith W, Kettner M (2019) [Diagnosis and treatment of glomus tumors of the skull base and neck]. Der Radiologe 59 (12):1051-1057. doi:10.1007/s00117-019-00605-0

6. Greenberg MS (2016) Handbook of Neurosurgery. Thieme,

7. Ehret F, Kufeld M, Fürweger C, Haidenberger A, Schichor C, Tonn JC, Muacevic A, Hempel JM (2020) Single-session image-guided robotic radiosurgery and quality of life for glomus jugulare tumors. Head \& neck 42 (9):2421-2430. doi:10.1002/hed.26231

8. Ehret F, Kufeld M, Fürweger C, Haidenberger A, Schichor C, Lehrke R, Fichte S, Senger C, Bleif M, Rueß D, Ruge M, Tonn JC, Muacevic A, Hempel JM (2020) Image-guided robotic radiosurgery for glomus jugulare tumors-Multicenter experience and review of the literature. Head \& neck.

doi:10.1002/hed.26439

9. Fisch U (1982) Infratemporal fossa approach for glomus tumors of the temporal bone. The Annals of otology, rhinology, and laryngology 91 (5 Pt 1):474-479. doi:10.1177/000348948209100502

10. Patel NS, Carlson ML, Pollock BE, Driscoll CLW, Neff BA, Foote RL, Lohse CM, Link MJ (2018) Longterm tumor control following stereotactic radiosurgery for jugular paraganglioma using 3D volumetric segmentation. J Neurosurg:1-9. doi:10.3171/2017.10.Jns17764

11. Wakefield DV, Venable GT, VanderWalde NA, Michael LM, 2nd, Sorenson JM, Robertson JH, Cunninghan D, Ballo MT (2017) Comparative Neurologic Outcomes of Salvage and Definitive Gamma Knife Radiosurgery for Glomus Jugulare: A 20-Year Experience. Journal of neurological surgery Part B, Skull base 78 (3):251-255. doi:10.1055/s-0036-1597986

12. Hafez RFA, Morgan MS, Fahmy OM, Hassan HT (2018) Long-term effectiveness and safety of stereotactic gamma knife surgery as a primary sole treatment in the management of glomus jagulare tumor. Clinical neurology and neurosurgery 168:34-37. doi:10.1016/j.clineuro.2018.02.037

13. Gulya AJ (1993) The glomus tumor and its biology. The Laryngoscope 103 (11 Pt 2 Suppl 60):7-15. doi:10.1002/lary.1993.103.s60.7

14. Sallabanda K, Barrientos H, Isernia Romero DA, Vargas C, Gutierrez Diaz JA, Peraza C, Rivin Del Campo E, Praena-Fernandez JM, López-Guerra JL (2018) Long-term outcomes after radiosurgery for glomus jugulare tumors. Tumori 104 (4):300-306. doi:10.1177/0300891618765576

15. Lim M, Bower R, Nangiana JS, Adler JR, Chang SD (2007) Radiosurgery for glomus jugulare tumors. Technology in cancer research \& treatment 6 (5):419-423. doi:10.1177/153303460700600507

16. Suárez C, Rodrigo JP, Bödeker CC, Llorente JL, Silver CE, Jansen JC, Takes RP, Strojan P, Pellitteri PK, Rinaldo A, Mendenhall WM, Ferlito A (2013) Jugular and vagal paragangliomas: Systematic study of management with surgery and radiotherapy. Head \& neck 35 (8):1195-1204. doi:10.1002/hed.22976

17. Ivan ME, Sughrue ME, Clark AJ, Kane AJ, Aranda D, Barani IJ, Parsa AT (2011) A meta-analysis of tumor control rates and treatment-related morbidity for patients with glomus jugulare tumors. $J$ Neurosurg 114 (5):1299-1305. doi:10.3171/2010.9.Jns10699 
18. Prasad SC, Mimoune HA, D'Orazio F, Medina M, Bacciu A, Mariani-Costantini R, Piazza P, Sanna M (2014) The role of wait-and-scan and the efficacy of radiotherapy in the treatment of temporal bone paragangliomas. Otology \& neurotology : official publication of the American Otological Society, American Neurotology Society [and] European Academy of Otology and Neurotology 35 (5):922-931. doi:10.1097/mao.0000000000000386

19. Lieberson RE, Adler JR, Soltys SG, Choi C, Gibbs IC, Chang SD (2012) Stereotactic radiosurgery as the primary treatment for new and recurrent paragangliomas: is open surgical resection still the treatment of choice? World Neurosurg 77 (5-6):745-761. doi:10.1016/j.wneu.2011.03.026

\section{Figures}
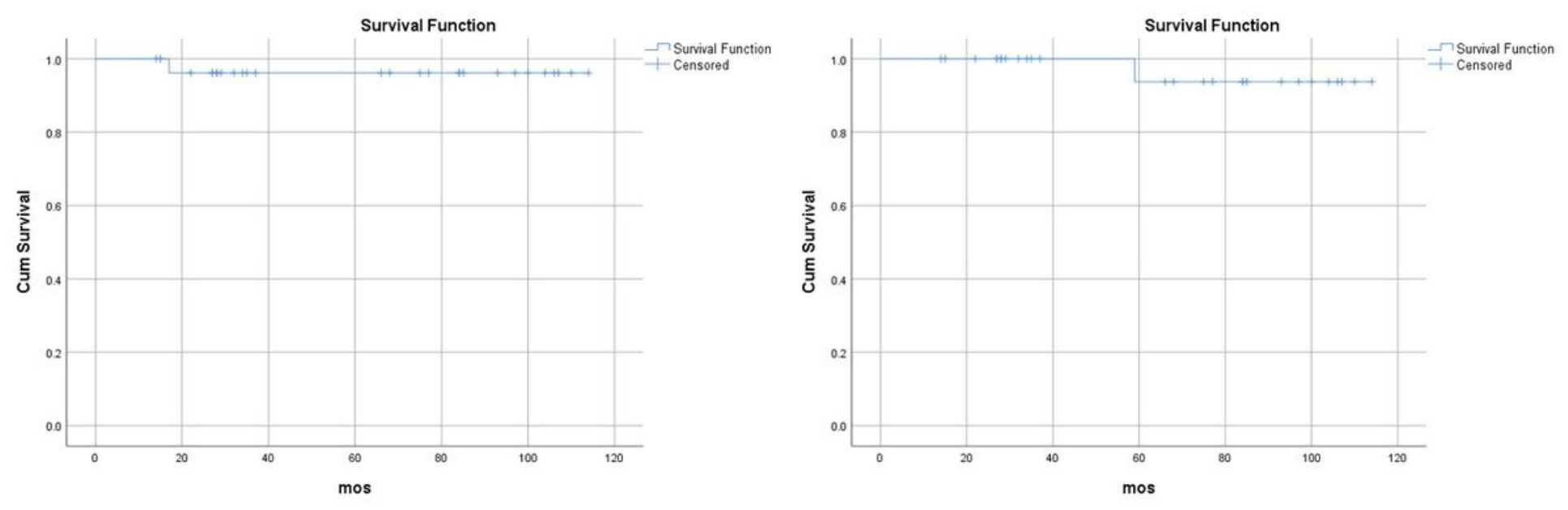

\section{Figure 1}

. Progression-free survival (left) and overall survival (right) of our study cohort in the median follow-up period of 66 months. 

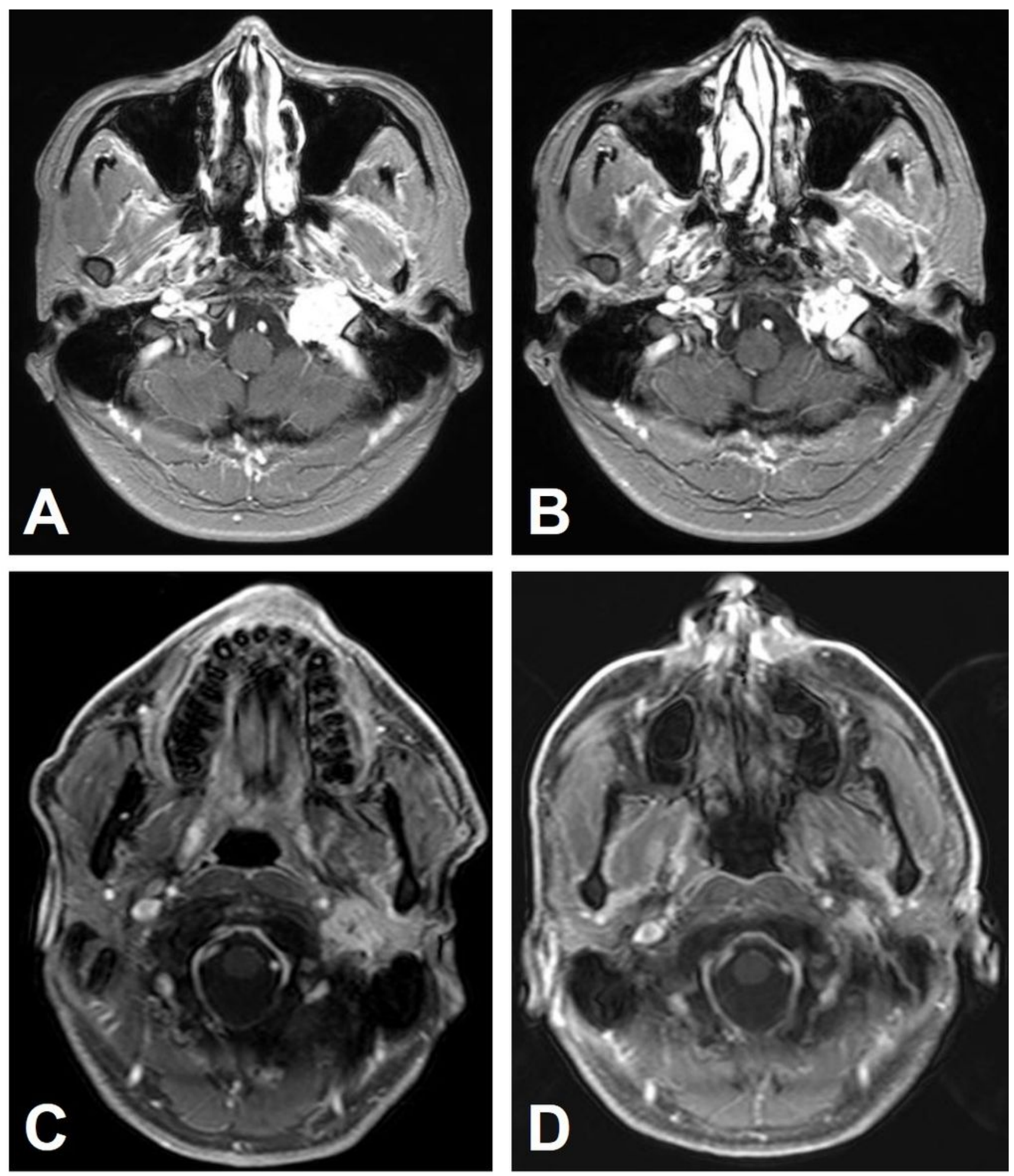

Figure 2

A, 35-year-old male presented with left tongue deviation and dysphagia. We performed CyberKnife therapy (CKT) with the prescribed dose covering 95\% of the planning target volume (D95) of 2100cGy in 3 fractions for target tumor volume of $10 \mathrm{cc}$. B, Follow-up MRI at 100 months after CKT showed reduced tumor size. C, 41-year-old male presented with left pulsatile tinnitus and sensory neural hearing 
impairment. D, We performed CKT with D95 of 2400cGy in 3 fractions for target tumor volume of $18 \mathrm{cc}$. Follow-up MRI at 75 months after CKT showed reduced tumor size.

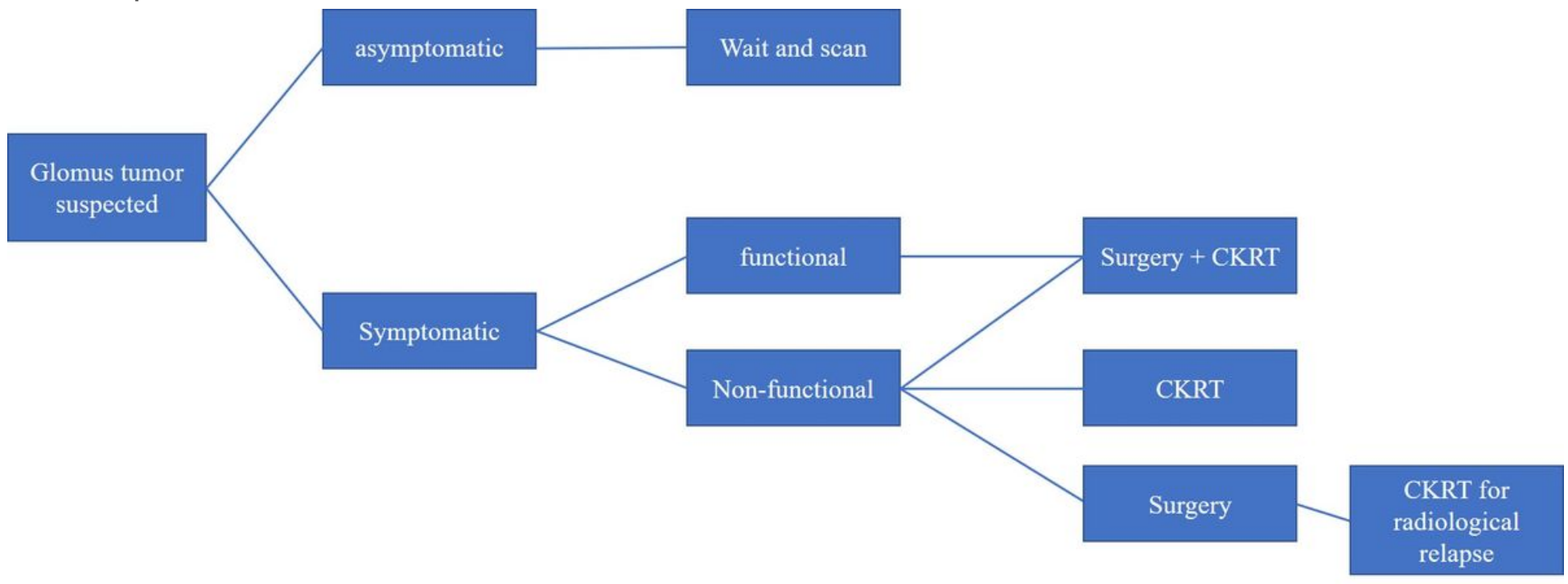

\section{Figure 3}

A flow-chart we suggest based on our experience. The management of head and neck paragangliomas is decided based on its presence of symptoms and the status of hormone production. We should treat symptomatic non-functional paragangliomas by upfront CyberKnife therapy (CKT). 